\title{
Las prácticas psicológicas en el ámbito escolar y su relación con el consumo de psicofármacos en \\ la niñez
}

\author{
Psychological practices within school context in \\ relation to pharmaceuticals consumption
}

\section{Práticas psicológicas no ambiente escolar e sua relação com o consumo de drogas psicotrópicas na infância}

\author{
Pablo Chouhy Algorta \\ ORCID ID: 0000-0002-3048-5788 \\ Universidad de la República, Uruguay \\ Autor referente: pablitochouhy@gmail.com \\ Historia editorial \\ Recibido: 19/03/2018 \\ Aceptado: 26/03/2019
}

\section{RESUMEN}

El presente artículo pretende poner de relieve algunos resultados de la investigación "Las prácticas psicológicas en el ámbito escolar ante el fenómeno de la medicalización de la infancia". Dicha investigación, se apuntaló para llevar a cabo el trabajo analítico en desarrollos conceptuales en torno al fenómeno de la medicalización de las subjetividades infantiles contemporáneas, así como en algunos estudios sobre las prácticas "psi" enmarcadas en lo que Michel Foucault denominó como "gubernamentalidad". (Foucault, 2006). La investigación realizada fue de corte cualitativo y el método orientador del análisis y aproximación al campo fue la

Teoría Fundamentada. Asimismo, la técnica privilegiada para recabar datos fue la entrevista en profundidad, entrevistándose a psicólogos que trabajasen en el ámbito escolar en la ciudad de Montevideo y zona metropolitana; tanto en el sub-sistema público como en el privado.

En este artículo se destacan los resultados vinculados a lo estrictamente farmacológico. Los psicólogos del ámbito educativo visualizan tendencias a brindar una respuesta basada en lo farmacológico dentro del sistema educativo, encarnadas en algunas maestras. Como consecuencia, llevan adelante una gran cantidad de acciones 
trabajando con estas sobre los sentidos y lógicas de este fenómeno, en pos de lograr que la institución escolar dé una respuesta más asertiva. Mantienen preocupaciones en torno al abuso de psicofármacos en la niñez, pero intentan no tener un posicionamiento moral ante la posibilidad del uso de dichos fármacos. Se entiende que llevan adelante una "psicologización" (Rose, 1996) de la problemática, lo cual colisiona con las perspectivas más tributarias de las neurociencias.

Palabras claves: Psicofármacos; prácticas psicológicas; gubernamentalidad

\section{ABSTRACT}

This paper advances some results from the research "Psychological practices within schools in the context of the medicalization of childhood". Said research built on recent work based on Foucault's notion of "governmentality" (Foucault, 2006) to conceptualize child subjectivities and the so-called "psi" practices.

Research methods drew on a qualitative research strategy based on the approach and guidelines of Grounded Theory's. Data was collected through in-depth interviews with psychologists who worked in schools in the city of Montevideo and metropolitan area, both in the public and private subsystems.

The results discussed in this paper focus primarily on pharmacological issues. Psychologists working within school contexts are concerned about teachers' tendency to give pharmacological response to students' behavioral problems. Consequently, they develop myriad actions of interventions with teachers, working with the latter on the meanings and logics of this phenomenon. Psychologists' goal is to achieve a more assertive response by educational establishments. They do not take a moral stance regarding psychopharmaceutical consumption in childhood, though they worry about abuse. They deem these practices as conducive to "psychologization" (Rose, 1996), which collides with neuroscientific approaches.

Keywords: Psychopharmaceuticals; psychological practice; governmentality

\section{RESUMO}

Este artigo tem como objetivo destacar alguns resultados da pesquisa "Práticas psicológicas no ambiente escolar diante do fenômeno da medicalização da infância". Esta pesquisa teve como objetivo realizar o trabalho analítico em desenvolvimentos conceituais em torno do fenômeno da medicalização das subjetividades das crianças contemporâneas, bem como em alguns estudos sobre práticas "psi" enquadradas no que Michel Foucault denominou "governamentalidade" (Foucault, 2006).

A pesquisa realizada foi qualitativa e 0 método norteador da análise $e$ abordagem ao campo foi a Teoría Fundamentada nos Dados. Da mesma forma, a técnica privilegiada para coleta de dados foi a entrevista em profundidade, entrevistando psicólogos que atuavam no ambiente escolar na cidade de Montevidéu e na região 
metropolitana; tanto nos subsistemas públicos como privados.

Este artigo destaca os resultados ligados ao estritamente farmacológico.

Os psicólogos do campo educacional visualizam tendências para oferecer uma resposta baseada no farmacológico dentro do sistema educativo, encarnadas em alguns professores. Como resultado, eles realizam uma série de ações que trabalham com estas tendências ao longo dos sentidos e lógica desse fenômeno, a fim de fazer com que a instituição escolar dê uma resposta mais assertiva. Eles mantêm preocupações sobre o abuso de drogas psicoativas na infância, mas tentam não ter uma posição moral diante da possibilidade de usar tais drogas. Entende-se que eles realizam uma "psicologização" (Rose, 1996) do problema, que colide com as perspectivas mais tributárias das neurociências.

Palavras-chave: Psicofármacos; práticas psicológicas; governamentalidade

n el presente artículo se pretende exponer conocimiento producido a partir de la
investigación "Las prácticas psicológicas en el ámbito escolar ante el fenómeno de la medicalización de la infancia"; específicamente los resultados relacionados a la problemática del consumo de psicofármacos. Para llevar a cabo el trabajo analítico se utilizaron desarrollos conceptuales en torno al fenómeno de la medicalización de las subjetividades infantiles contemporáneas y de las prácticas "psi", enmarcadas en lo que Michel Foucault (2006) denominó como "gubernamentalidad".

Desde la segunda mitad del siglo XX asistimos a un incremento sustantivo de la "detección" de síntomas y cuadros psicopatológicos en la infancia escolarizada, con una consecuente prescripción terapéutica basada en el consumo de psicofármacos. El caso paradigmático ha sido el de los síntomas de la desatención, la hiperactividad y la impulsividad englobados frecuentemente bajo la entidad psicopatológica de "Trastorno por déficit atencional con o sin hiperactividad" (TDA, TDAH, ADD o ADHD por sus siglas en español e inglés); y tratados terapéuticamente en base al suministro de clorhidrato de metilfenidato (más conocido como "Ritalina" por su nombre comercial). Ante tal fenómeno, varios investigadores y profesionales de las Ciencias Sociales, el ámbito médico y el campo "Psi", han alertado acerca del sobrediagnóstico de tal 
cuadro, y la tendencia actual de visualizar cualquier problema o manifestación de conflicto dentro del ámbito educativo como reflejo de un cuadro psicopatológico.

Se considera por demás pertinente visibilizar el accionar de la Psicología en el ámbito educativo ante estas situaciones, en tanto es una disciplina cuyo saber y accionar se encuentra directamente comprometido e interpelado por tal problemática, pero que sin embargo ha estado ausente en los estudios sobre la temática; al menos a nivel nacional. Estos últimos, se han ocupado más que nada del análisis de las instituciones médicas y de las educativas, así como de los actores más "tradicionales" de las mismas (psiquiatras, pediatras, neurólogos, maestros, directores, etc.).

\section{Metodología}

Es importante mencionar que la investigación se planteó un enfoque cualitativo de modo de poder acceder al universo de las prácticas psicológicas y sus sentidos, contemplando al fenómeno en su singularidad, su complejidad y sus contextos específicos (Vasilachis, 2006). Cuando se escogen métodos predominantemente cualitativos, se está optando por hacer énfasis en la comprensión e interpretación de los fenómenos, así como por dejar de lado la ilusión de develar las relaciones causaefecto, para, en cambio, interesarse por distintos universos simbólicos y tramas de significado (Apud, 2013).

El método orientador del análisis y aproximación al campo fue la Teoría Fundamentada (TF), congruente con la finalidad de construir teoría a partir de los datos recabados, y no de comprobar o contrastar desarrollos teóricos pre-existentes. Estos sí sirvieron, ni más ni menos, que como soportes para el trabajo analítico. Así, cobraron especial importancia los procesos de categorización que se llevan a cabo a partir de los datos recabados, asumió más relevancia el carácter inductivista de la investigación, y orientó la investigación la finalidad de producir teoría sustantiva enraizada en lo que se toma del campo (Soneira, 2006). A su vez, privilegiando la 
entrevista en profundidad como método de recolección de datos, se entrevistó a psicólogos que trabajan en el ámbito escolar en la ciudad de Montevideo y zona metropolitana, tanto en el sistema público como en el privado. Las entrevistas se realizaron entre agosto y diciembre del 2017, valiéndose de los criterios de accesibilidad, heterogeneidad y saturación (Valles, 1997).

\section{Referentes conceptuales}

\section{Medicalización de las infancias contemporáneas}

La medicalización de las sociedades es un proceso insoslayable de las sociedades contemporáneas estudiado por varios autores, dentro del cual la infancia es un blanco privilegiado sobre el que se despliegan múltiples prácticas. Si bien es posible encontrar múltiples definiciones de este fenómeno por parte de diversos autores, todos hacen alusión al pasaje de problemas cotidianos del existir humano a la órbita del accionar médico u otras profesiones afines del campo de la salud (Germain, 2007). En su genealogización del fenómeno, Foucault (2008) afirma que en el siglo XX la expansión de la medicina y sus lógicas es tal, que no parece tener campo exterior: ningún ámbito de la existencia humana parece quedar por fuera de su intervención. Ahora bien, Los procesos de medicalización se han visto, desde las últimas décadas del siglo XX hasta la actualidad, metamorfoseados por la brutal expansión de la prolífica industria farmacéutica y la reestructuración capitalista que pautaron una marcada mercantilización de la salud. Como efecto, se tornó muy evidente que la medicalización como fenómeno político podía rebasar la intervención técnicaprofesional del médico, pasando a ser la esencia de sus prácticas el consumo de fármacos (con la mediatización o no de un agente de la salud). Por lo tanto, el efecto es que la medicalización se parece cada vez más a una "medicamentalización" (Faraone, Barcala, Bianchi \& Torriccelli, 2009) o a una "farmaceuticalización" (Abraham, 2010), en tanto el mandato por el cuidado de la salud se equipara al 
consumo de fármacos, siendo el marketing y el lobby de la industria farmacéutica fundamental para explicar este proceso. Queda entonces planteado el hecho de que la intervención médica (e incluso a veces prescindiendo de esta) queda en la actualidad prácticamente homologada a la prescripción de fármacos (Stolkiner, 2012), lo que genera que la medicalización esté pautada por la "farmacolización" y "sobrefarmacolización". Como impacto en la esfera subjetiva, se puede decir que impera actualmente la lógica de erradicar el malestar a través de soluciones fáciles y rápidas. Se hace referencia a los cambios en las nuevas formaciones subjetivas mayormente vinculadas a la instantaneidad, el mandato de felicidad constante y de ausencia del dolor, la valorización de la salud y el fomento al consumo (Pinafi, 2013). Estas líneas subjetivantes se retroalimentan y son indisociables de las lógicas de la industria farmacéutica que permean y diagraman cada vez más el fenómeno de la medicalización, lo que sedimenta el terreno para el consumo de fármacos como objetos-fetiche.

En el campo de la infancia, el proceso medicalizador actual no está regido entonces solamente por los movimientos patologizadores y normalizantes tradicionales de los niños, niñas y adolescentes (NNA), sino también por la preponderancia cada vez mayor de las herramientas psicofarmacológicas como respuesta privilegiada. Se puede afirmar que la infancia es uno de los territorios en donde más se despliega el fenómeno de la medicalización, con algunas características propias. Así, podemos centrarnos en dos críticas que frecuentemente se realizan por parte de quienes estudian y teorizan sobre este tema: la tendencia a patologizar lo anómalo en el niño, realizando un perjudicial uso de la estrategia diagnóstica, y la predisposición a asumir rápidamente y sin evidencias suficientes de que la respuesta a tal problema pasará por una terapéutica erigida sobre el consumo de psicofármacos por parte del niño. Es por demás ilustrativo el aporte de Stolkiner (2012) cuando entiende que en el campo del sufrimiento infantil existe una operación que consta de un triple movimiento: 
"individualizar el proceso de salud-enfermedad-cuidado, reducir el padecimiento psíquico a la psicopatología y ontologizar luego el cuadro psicopatológico" (p.35).

\section{Prácticas Psicológicas a la luz de la gubernamentalidad}

El ya mencionado filosofo Michel Foucault introdujo el término "gubernamentalidad" en el marco de sus seminarios en el College de France en el año 1978. Foucault se abocó a estudiar nuevas formas de manejar las conductas que lo llevaran a las distintas prácticas de gobierno y su relación con el Estado (más específicamente con la razón de Estado) (Foucault, 2006). Así, el concepto de "gubernamentalidad" se encuentra muy vinculado a los procesos de conducción de las conductas del cuerpo poblacional, al decir de Chávez Bidart (2016): "refieren a las múltiples maneras en que diversos saberes han sido utilizados en la conducción de la población a través de cálculos, procedimientos y tácticas, y que tuvieron como consecuencia el nacimiento del Estado moderno" (p. 23).

Ahora bien, para enfocarse particularmente en las prácticas psicológicas, es muy conveniente centrarse en los estudios de la gubernamentalidad, pero de la forma que lo realizan los denominados "anglofoucaultianos" (Mussetta, 2009), y particularmente la figura de Nikolas Rose. Se comprende desde esta línea de investigación que en la propuesta foucaultiana hay una invitación metodológica a analizar las prácticas en su singularidad contextual e histórica, siempre teniendo en cuenta que las mismas obedecen a cierto tipo de racionalidad y lógicas que son más bien inmanentes y para nada universalizables (Castro-Gómez, 2010).

Las prácticas psicológicas, adquieren relevancia en tanto pueden ser pensadas como prácticas de gobierno que pretenden dirigir las conductas de los sujetos (Rose, 1996). Pero tal direccionamiento, no se realiza mediante la coacción, sino buscando la apropiación por parte de los sujetos de las mismas racionalidades y finalidades que articulan las prácticas de gobierno. A su vez, Rose vincula el desarrollo de la 
Psicología con los problemas de la ética, la política y el conocimiento, afirmando que el desarrollo de las sociedades occidentales es totalmente indisociable de la construcción de un conocimiento experto sobre el "yo", que redunda en procedimientos y técnicas orientadas a moldear, regular y administrar este objeto construido por el saber psicológico y dominios de saber afines. Rose afirma que en el proceso de disciplinarización de los conocimientos psicológicos, cada vez más esferas de la vida social fueron siendo pensados bajo la matriz de los saberes psicológicos, en auge desde fines del siglo XIX. Es una especie de progresiva "psicologización" de la vida cotidiana y social (algo relacionado, pero diferente a los procesos de medicalización). Sin embargo, Rose hace alusión en sus últimos trabajos sobre como el territorio ganado por las prácticas psicológicas de individualizar al sujeto para promover su autogobierno mediante la suposición de una interioridad propiamente psicológica, está perdiendo terreno ante los avances de las lógicas provenientes de las neurociencias en el siglo XXI. De esta forma, identifica en la actualidad como se perciben y problematizan los fenómenos que otrora eran concebidos dentro de la esfera psicológica en términos biomédicos, refiriéndose a este hecho como "achatamiento de la interioridad" o "somatización"; y aventurando el concepto de "yo neuroquímico" (Córdoba, 2012).

En esta línea, vale mencionar el contexto de "refundación" de la Psiquiatría a partir de los avances científicos en el campo de la neurobiología. La Psiquiatría tuvo un nuevo auge y se reconfiguró en sus intentos de tratar el sufrimiento mental como algo objetivable, natural y de base orgánica con dos fenómenos indisociables entre sí: los avances de las investigaciones neurobiológicas y el crecimiento de la industria farmacéutica. Ya desde finales del siglo XIX, se puso en cuestión la búsqueda por parte del positivismo científico de un correlato entre los fenómenos físico-anatómicos del cerebro y la totalidad de los fenómenos mentales. De esta manera, se dio lugar a una brecha entre el funcionamiento del cerebro y el funcionamiento mental y de la 
conducta humana, lo cual permitiría el advenimiento de una multiplicidad de saberes y teorías sobre lo mental (Pérez, 2010). Pero más allá de este campo que se abrió para un estudio de lo mental como algo que rebasaba el funcionamiento del cerebro, se continuaron estudios que para estudiar lo mental realizaban una jerarquización del funcionamiento cerebral por sobre otros factores. Los mismos tuvieron un nuevo auge en la última década del siglo pasado Este fenómeno es eminentemente político, fomentado por intereses corporativos (sobre todo de la industria farmacéutica), y ha generado "una clara ofensiva del paradigma científico positivista, que, en este campo, busca subordinar los fenómenos mentales y psicológicos a las lógicas del cerebro y sus métodos de investigación" (Pérez, 2010, p. 15).

Sin embargo, numerosos estudios por fuera del ámbito de las neurociencias dan cuenta de la imposibilidad de comprender linealmente las relaciones entre el funcionamiento cerebral y los procesos mentales, procesos que integran a un sujeto en toda su complejidad, como un ser social e histórico, determinado por el mundo simbólico en el cual se inscribe. Con este escenario se ponen de relieve dos modalidades muy distintas de comprender y operar sobre el sufrimiento mental.

\section{Resultados}

A continuación, se presentan algunos resultados de la investigación vinculados a las prácticas psicológicas referidas a la medicalización, pero haciendo foco mayormente en lo farmacológico y las prácticas que se realizan dirigidas a la interna del dispositivo escolar. Tales resultados son en función de la producción discursiva de los psicólogos entrevistados en la investigación mencionada.

\section{Presiones sobre lo farmacológico a la interna de la escuela}

Se hace referencia a la idea repetida de los actores entrevistados de que, más allá de sus acciones, la prescripción de medicación se determina en al ámbito de la salud, 
teniendo sus prácticas poca incidencia en esta decisión tomada por otro profesional (médico psiquiatra) en otro ámbito (ámbito de la salud, proceso terapéutico). Aluden a lo alejado que está el dispositivo de intervención psicológico de influenciar en estas dimensiones, incluso con un explícito "Nosotros no medicamos" (sub-sistema público (Pu.)). Asimismo, aluden a que no toman demasiado en cuenta si un niño está medicado o no para realizar sus intervenciones que consideran de índole institucional. Se vislumbra un territorio disciplinar ganado por la Psiquiatría como saber que se ha erigido como el idóneo para sancionar sobre esta problemática. Asimismo, el dominio de la Psiquiatría aparece de a momentos como un territorio hermético y de difícil acceso. Incluso, como un lugar de experticia con el cual no se debe interferir ni cuestionar: "Si hay un psiquiatra que indicó la medicación, yo no soy psiquiatra, la medicación se tiene que tomar" (sub-sistema privado (Pr.)).

A la interna de la escuela, aparecen destacados pedidos (explícitos en su mayoría) de las maestras de que ciertos niños tendrían que estar medicados, “...todos los años se da de que el maestro viene y te dice "este niño hay que medicarlo, hay que hacer algo" (Pu.) A veces, también mencionan decisiones de las direcciones de las cuales los psicólogos se enteran a posteriori. Este pedido de medicalización que se encarna en las maestras, lo viven como una visión reduccionista de la problemática, emergente de un sistema que se encuentra desbordado. Por lo tanto, suelen atender tal pedido, pero poniéndole un freno. Incluso la resistencia a estas perspectivas puede ser más intensa y despertar enojo hacia el enfoque que sostienen algunas maestras. Los entrevistados refieren a cómo gran parte de las acciones realizadas desde su rol es el trabajo con otros actores de la institución escolar, sobre todo con las maestras; erigiendo en ocasiones la articulación con el cuerpo docente como el quehacer primordial del psicólogo del ámbito educativo. En los discursos se hace patente que los psicólogos se abocan con intensidad a trabajar con las maestras y realizan esfuerzos para establecer un vínculo de trabajo colaborativo y complementario. Sin embargo, no 
siempre se logra. Una de las claras aspiraciones de los psicólogos en su trabajo con las maestras es re-situarlas en su posicionamiento sobrediagnosticador y promotor de lo farmacológico; tarea tan prioritaria como difícil. De esta manera, las intervenciones están vinculadas a generar sentidos sobre hechos, abrir visibilidad sobre fenómenos y promover nuevos enfoques, racionalidades y acciones por parte de otros actores.

Estos hechos advierten a los psicólogos sobre la posibilidad de que se esté pensando la problemática de los niños de una manera un tanto reduccionista, por lo que este enfoque y lógica de medicar lo que se desvía puede pasar a ser un objeto de intervención para estos psicólogos Aquí parecen subyacer dos concepciones distintas del sufrimiento y el malestar humano. Por un lado, los psicólogos presentan una visión dinámica y contextual del sufrimiento, haciendo énfasis en indagar las condiciones en las cuales el mismo se produce, así como trayendo la importancia de tener una mirada evolutiva. Por otro lado, otros actores (los más señalados suelen ser algunas maestras) muestran su predisposición a adherir con mayor facilidad a acciones que evidencian el entendimiento de muchos malestares humanos como desordenes bioquímicos del cerebro. Estas concepciones, tributarias de las ciencias biológicas, predisponen al pedido de medicación como única respuesta válida a diversas manifestaciones del malestar psíquico: “...Que cuesta. Cuesta porque a veces las maestras necesitan el diagnóstico y las pastillas para sentirse tranquilas que algo está pasando, que está en tratamiento. Estos otros tratamientos que son menos visibles les cuestan el tiempo de espera." (Pr.).

Asimismo, se percibe impotencia ante la permeabilidad que puede tener la industria farmacéutica para influenciar en las lógicas del sistema educativo, visualizándolo como un grupo de poder económico que influye no solamente en el ámbito de la salud, sino también en el educativo, con lógicas lucrativas y de marketing: "Entonces, jojo con eso! Porque esto no deja de ser por plata. Las pastillas no te las regalan, las fabrican, las distribuyen, etc. Todo por plata." (Pu.). 


\section{Adecuar la escuela}

Muchas veces, las prácticas mencionadas son reconocidas en su potencial de evitar un tipo de tratamiento de índole farmacológico, o de complementarlo y desmitificarlo en las representaciones sociales "mágicas" que suelen asociársele; así como también involucrar a adultos y señalar que el problema no está solo en el niño. Por tanto, coinciden en realizar intervenciones que incluyan al niño señalado como problemático, pero rebasando al mismo. Por ende, sus acciones irán dirigidas a hacer lo posible por generar cambios en el entorno educativo y facilitar el aprendizaje, evitando intervenciones que se basen únicamente en corregir y adaptar al niño a la institución. Se busca en cambio (otra vez bastante consciente y explícitamente) que la institución pueda adaptarse al niño en pos de promover sus potencialidades y hacer valer su singularidad: "Para mí, el rol del ámbito educativo es que el chiquilín tenga la educación más digna posible y si el chiquilín necesita determinadas adaptaciones o estímulos particulares la escuela se lo tendría que brindar porque es el transito curricular del chiquilín y de su vida." (Pu.).

Aquí, la idea subyacente que parecen compartir los psicólogos es que en la institución educativa se patologizan las conductas desviadas: "la escuela tiende a ser normalizadora históricamente como institución, se puede volver prescriptiva sobre algo que no le corresponde. Por ejemplo, que el niño tome medicación." (Pu.). Los entrevistados concuerdan en identificar un desacople entre el funcionamiento de la institución escolar y las formas subjetivas de la infancia contemporánea. A su vez, se preguntan si el sobrediagnóstico (y la consecuente sobremedicalización que suele conllevar) no se explican por esta disonancia entre una institución que acoge a los niños cada vez más horas, exigiéndoles atención y quedarse sentados, cuando sus procesos de socialización y producción subjetiva se han modificado drásticamente, sobre todo con respecto a las imágenes y la movilidad. 
Es que las producciones subjetivas de la infancia contemporáneas están compuestas por procesos muy distintos en relación a pocos años atrás. De esta manera, el acceso a la información, la importancia del mundo de lo visual, la vertiginosidad de lo temporal y los vínculos modificados (más de pares) con los adultos, producen formas subjetivas en la infancia novedosas, propias de estas generaciones y alejadas de la subjetividad infantil propia de la modernidad. Estas nuevas subjetividades, no se ven acompasadas por los distintos territorios existenciales por los que circula la niñez, y menos aún por el sistema escolar (Corea y Lewkowicz, 1999), al cual los psicólogos entrevistados reconocen como una estructura bastante rígida y con una inercia considerable a seguir funcionando igual al momento en la cual fue creada. Como consecuencia de tal desacople, muchas conductas tradicionalmente inesperables irrumpen en el espacio escolar. Como explica Untoiglich (2014) cuando denuncia que la "medicamentalización" (medicalización, pero vinculada estrechamente al consumo de fármacos) de la infancia está persiguiendo como fin "controlar las conductas de los niños y adaptarlos a un sistema escolar, que en su estructura central no ha variado en los últimos tres siglos. Continuamos educando con métodos del siglo XIX a niños del siglo XXI”. (p. 26).

Se hace evidente en las afirmaciones de los psicólogos entrevistados, cómo la Psicología tiene momentos de corte con su tradición de auxiliar el poder disciplinario más clásico: aquel que se ejercía sobre el cuerpo individual en las instituciones disciplinarias, modelando los comportamientos y castigando el desvío a la norma; en definitiva, la anatomopolítica (Foucault, 2006). Pero no debe leerse aquí que las racionalidades de las prácticas psicológicas están eximidas de los juegos de poder, algo que sería inconcebible. Con la noción de gubernamentalidad (Foucault, 2006), se puede comprender cómo el biopoder es el ejercicio de poder privilegiado en la contemporaneidad. La forma de regular la vida de las poblaciones, supondrá entonces, potenciar los rasgos vitales y apelar al autoconocimiento, autorregulación y explotación 
de las potencialidades de los sujetos. A su vez, se fomenta la emergencia de las singularidades y la aceptación de la diferencia en distintos ámbitos de la vida social (en este caso, el ámbito educativo) dentro de cierto marco normativo, en un juego de libertades cada vez más posible en el escenario de la hegemonía ideológica neoliberal. (Rose, 2007).

\section{Sentidos sobre lo farmacológico}

Los participantes de la investigación, reconocen el consumo de psicofármacos en la niñez como una problemática muy acuciante a atender en sus prácticas, y que tiene sus manifestaciones en el sistema educativo. El fenómeno siempre aparece inseparable y como resultado del sobrediagnóstico al que se somete la niñez contemporánea, y de los desfasajes mencionados entre funcionamiento de las instituciones educativas y las producciones subjetivas contemporáneas. Prácticamente todos los entrevistados espontáneamente plantean que se interrogan y se llaman a la reflexión sobre la dimensión de la problemática de la farmacolización de la infancia y su estrecho vínculo con el rol que juega el sistema educativo en el desarrollo vital de los niños. Estas interrogantes que se plantean indican que el tema en cuestión es de importancia para los psicólogos del ámbito educativo, y se lo plantean en términos tanto cualitativos como cuantitativos.

El ensayo de respuesta a la pregunta que ellos mismos se formulan, difiere bastante entre el sub-sistema público y los colegios privados. En el primero, se reconoce el fenómeno en una suerte de incontrolable expansión. Los psicólogos se preocupan por un hecho que dan por sentado como el aumento de la niñez medicada: “...estoy convencida que está sobremedicalizada la niñez" (Pu.). Identifican que el sentido preponderante que cobra el consumo del fármaco es la respuesta rápida y "mágica" a producciones subjetivas de la infancia contemporánea que irrumpe con problemas conductuales y de aprendizaje en el sistema educativo y que desborda las redes de 
sostén familiar que, frecuentemente, son muy magras. Por tanto, este fenómeno es percibido como in crescendo y un tanto fuera de control. Se hace patente que los psicólogos vivencian el fenómeno con preocupación, impotencia y cierto enojo.

Por su parte, en los colegios privados se comparten las visiones sobre el lugar que viene a ocupar el consumo de psicofármacos en la relación del niño con el sistema educativo. Pero, se diferencian los discursos en lo concerniente al desarrollo del fenómeno, al cual consideran en retracción. Si bien se coincide en identificar el fenómeno de la medicalización de la infancia (con un consumo abusivo de psicofármacos) por parte de la niñez escolarizada, se estima que este proceso tuvo su auge y su "techo" en cuanto expansión alrededor de 5 años atrás. La difusión crítica (prácticamente en modalidad de denuncia) académica y mediática sobre el fenómeno habría generado que los actores involucrados se muevan con más cautela y sensibilidad ante el problema, evitando medicar como "acto reflejo". Esto abarca tanto a actores del sistema educativo (maestras) como del sector salud (psiquiatras) quienes habrían cambiado un tanto su postura a pesar de las presiones de la industria farmacéutica: : “...yo creo que también varió porque hay una mirada mucho más atenta de la sociedad, a nivel de prensa, a nivel de medios de difusión, el tema ritalina ha estado muy sobre la mesa, entonces creo que esto también es como un aviso a los técnicos, a los Psiquiatras, a los neuropediatras. Es, bueno, antes de medicar vamos a estar absolutamente seguros que éste es el camino porque tenemos una sociedad que nos está denunciando, tenemos laboratorios que también están generando esa guerra de medicación, entonces creo que eso también ha hecho afinar a la hora de diagnosticar" (Pr.)

Por su parte, aparecen producciones discursivas que se enlazan señalando la frecuente idealización o demonización que generan otros actores con respecto al psicofármaco, en tanto objeto fetiche a ser consumido. Es más que nada alertado el fenómeno de la idealización de la utilización de la "pastilla", y varios entrevistados 
manifiestan cierto resquemor frente a la idea instalada en actores del ámbito educativo de que el consumo de psicofármacos suponga la solución definitiva y el final de la problemática.

Es pertinente destacar, que, si bien como se mencionó, los psicólogos se mantienen alerta ante el fenómeno del consumo abusivo de psicofármacos en la infancia, intentan mantener una posición desprejuiciada ante las situaciones que se les presentan. Es notorio entonces, cómo los psicólogos sostienen que el posicionamiento más asertivo ética y profesionalmente parece ser aquel que evalúa la pertinencia o no del fármaco según la singularidad de la situación y la información que se recaba de otros profesionales, familiares y docentes. Es decir, que se intenta no quedar atrapados en una postura moral de demonización del fármaco, así como dejan en claro que no idealizan sus efectos. Por el contrario, más allá de que puedan encontrarlo necesario siempre lo considerarán limitado en cuanto a sus logros: “...porque no nos va a salvar la pastilla. Ni tampoco nos va a hundir en el fango. Es una terapéutica si lo usas bien, yo he visto resultado en los niños" (Pu.).

Relacionado a lo antedicho, los psicólogos alertan sobre el problema de visualizar el consumo de psicofármacos desprovisto de otro tipo de cambios, movimientos o terapéuticas (sobre todo estrategias psicoterapéuticas) que incluyan otros actores y otras dimensiones de los malestares que aquejan a los niños. Se podría decir que les genera enojo e incredulidad, cómo en algunas familias o maestras subyace la idealización que se mencionaba anteriormente, llevando a considerar que con el consumo de fármacos se soluciona definitivamente una problemática que desde sus puntos de vista es mucho más compleja. Por tanto, coinciden en considerar al psicofármaco como una herramienta terapéutica más, que debe estar acompañada de otros movimientos y procesos de cambio. En general se refieren al acompañamiento y la intervención de otro profesional, pero también pueden hacer referencia a un intento de mayor entendimiento de la problemática por adultos que son interpelados por la 
misma, de cambio de posturas y hábitos de los adultos, de adecuación del sistema educativo a la realidad del niño, de acompañamiento en lo emocional a la situación del mismo, de trabajos a la interna de la dinámica familiar, de modificaciones del vínculo singular entre el docente y aquel niño rotulado como problemático. Pero siempre se acentúa la idea de que la medicación debe ir acompañada de otras terapéuticas y movimientos: "Nosotros insistimos en eso, que la medicación no es mágica, que es la medicación acompañada de otras cosas. Y a veces no es la medicación, a veces hacen falta otras cosas más desde la perspectiva psicológica y el apoyo familiar." (Pu.)

\section{Conclusiones}

Se puede aseverar que los psicólogos del ámbito educativo no se plantean interferir en el accionar de otros profesionales respecto al diagnóstico y la prescripción de psicofármacos. Tampoco se proponen oficiar como ese espacio terapéutico necesario para acompañar al fármaco, ya que se diferencian de la Psicología clínica.

Empero, sí se proponen intervenir sobre algunas lógicas medicalizadoras, así como realizar una buena coordinación con los profesionales tratantes (o postulantes a tratar la problemática) de modo que el diagnóstico sea lo más acertado posible, así como un eventual consumo de psicofármacos. La Psicología puede ser un agente funcional al proceso de medicalización, colocando diversas problemáticas en el terreno de la salud. Tal cual nos recuerda Untoiglich (2014) "Cuando la maquinaria patologizadora se instala, sobre todo en la infancia, puede estar incentivada por la medicina, pero también por la psicología e incluso cierto psicoanálisis" (p.25). Empero, puede decirse que se encuentra dentro de las prácticas de los psicólogos de las escuelas el propósito de interferir sobre ciertas lógicas y procederes propios del fenómeno de la medicalización de la infancia. En función del dispositivo que enmarca sus prácticas mayoritariamente como "intervenciones institucionales", el área en donde puede verse 
más gráficamente tal intervención sobre las lógicas medicalizadoras, es en el trabajo ya analizado a la interna de la escuela, específicamente con las maestras.

En esta esfera, se da algo bien interesante que puede ser interpretado como evitar en algunos casos la medicalización de problemáticas mediante una "psicologización". Es decir, aquello que Rose (1996) desarrolló sobre la expansión del lenguaje y mirada psicológica en varias esferas de lo social, instaurando la idea de una profundidad de un yo que debe ser comprendido, explorado e interpretado; es una lógica que se va imponiendo y diseminando, con distintos grados de intencionalidad, en las formas en que otros actores conciben la problemática: familias, maestras, etc. Parece encastrarse bien en las modalidades gubernamentales de las sociedades liberales avanzadas (Rose, 2007) que conducen las conductas de las poblaciones haciendo hincapié en la singularidad de las existencias y sus padecimientos, en la importancia de potencializar capacidades y de estimular la autogestión de los propios proyectos, del propio cuerpo, de la propia salud. Se identifica que esta "psicologización" puede en ocasiones complementarse, apuntalarse $o$ no interferir con el proceso de medicalización. Sin embargo, se hace también visible que las visiones que se promueven pueden colisionar con las explicaciones "bio" sobre los malestares humanos impulsadas por las neurociencias, y tomadas por la psiquiatría, así como por todo el cuerpo social (Pérez, 2010). Este parece ser una de las tensiones que predominan en las prácticas de los psicólogos del ámbito educativo y que gestionan con varios actores. Es decir, que la "psicologización" manifestada en las racionalidades de posicionarse ante el malestar humano haciendo emerger al sujeto sufriente con su contexto y singularidades, propiciar interrogantes en torno a lo sintomático y suponer una entidad (yo, psiquismo, etc.) dotada de cierta profundidad que se hace necesaria explorar e interpretar; se entiende como un movimiento muy distinto, y que puede amortiguar cierta inercia sobrediagnosticadora y sobremedicalizadora, al de comprender el malestar humano únicamente en términos 
de desórdenes bioquímicos en la esfera del cerebro, susceptibles su mayoría de un rápido reacomodo a través de la utilización de psicofármacos.

Por lo antedicho, se puede comprender la racionalidad de las prácticas psicológicas en torno a la medicalización como "post-disciplinaria", tributaria de las lógicas gubernamentales propias de las sociedades liberales avanzadas. En la misma, el manejo de la población escolarizada se realiza haciendo hincapié en fortalecer las potencialidades, singularidad y autonomía de los sujetos (niños) afectados, y no en adaptar el desvío o lo anómalo a la institución. Las prácticas psicológicas persiguen como una de sus finalidades destacadas modificar la institución escolar para incrementar su poder inclusivo y el bienestar de la comunidad educativa. Además, en la racionalidad de las prácticas psicológicas, influye una percepción de los psicólogos del ámbito educativo de la existencia de sobrediagnósticos y sobrefarmacolización en la niñez uruguaya. La misma los predispone a guiar sus acciones en una dirección alternativa a este fenómeno. Se puede afirmar que la racionalidad de las prácticas psicológicas conserva aún sus aristas de reproducir el circuito de la medicalización en el cual se encuentra comprometido el sistema educativo, pero mayoritariamente predomina una lógica de "frenar" un fenómeno que se percibe con preocupación y se intenta ir moderando (aparentemente con cierto éxito). No se está planteando en este artículo que los psicólogos se opongan a las intervenciones de profesionales de la salud o al consumo de psicofármacos por parte de los niños, ya que de hecho se constata que la posición de los psicólogos ante lo farmacológico es la de evitar la demonización y la idealización del mismo. Pero sí se mantienen alerta a cierto accionar automático y muy arraigado en el ámbito escolar que lleva a los sobrediagnósticos y a la sobrefarmacolización. Con respecto a la sobrefarmacolización, se mencionó que los psicólogos no se oponen al fármaco en sí mismo. Sin embargo, sí trabajan en pos de desterrar ciertas concepciones que colocan al fármaco como la respuesta privilegiada ante cualquier problema de la niñez 
escolarizada, erigiéndose además como la solución definitiva. Se hace hincapié en la necesidad de prescribir el fármaco únicamente en las situaciones que realmente lo ameriten, y no irreflexivamente como respuesta a la manifestación de aquello heterogéneo que interroga. Además, el fármaco es visto como una herramienta terapéutica más que siempre debe ser acompañada por otros movimientos o procesos terapéuticos.

Desde el autor del presente artículo, se entiende las prácticas psicológicas ante los sobrediagnósticos y la sobrefarmacolización como regidas por una racionalidad de "psicologizar" los fenómenos. Es decir, que se tiende a hacer foco en un sujeto y su contexto, dotado de una profundidad que debe ser asequible de ser interpretada para dar una respuesta atinada a las manifestaciones de las emociones y el sufrimiento humano. Tal idea subyacente colisiona con la tendencia impulsada por las neurociencias y la industria farmacéutica de comprender el padecimiento humano en términos de síndromes y enfermedades que se corresponden con fenómenos de base biológica; y de dar respuesta a tales padecimientos basándose en la prescripción de psicofármacos.

Esto genera que las prácticas psicológicas en el sistema educativo estén teniendo un efecto amortiguador del fenómeno de la medicalización. Si bien la Psicología funcionó históricamente, y continúa funcionando en ocasiones, como una disciplina auxiliar a la medicina, la "psicologización" de la problemática colisiona, tensiona e interpela los fundamentos en los cuales se erige la medicalización actual de la niñez contemporánea. Entonces, la Psicología muestra aquí no solamente tener un enfoque y accionar independiente de las disciplinas a las que históricamente se subordinó, sino también capacidad para promover estas perspectivas en otros actores involucrados en el fenómeno de la medicalización de las infancias (incluyendo a los propios niños). 


\section{Referencias}

Abraham, J. (2010). Pharmaceuticalization of society in context: theoretical, empirical and health dimensions. Sociology, 44(4), 603-622.

Apud, I. (2013). Repensar el Método Etnográfico. Hacia una Etnografía multitécnica, reflexiva y abierta al diálogo interdisciplinario. Antípodas. Revista de Antropología y Arqueología, (16), 213-235.

Castro-Gómez, S. (2010). Historia de la gubernamentalidad; razón de Estado, liberalismo y neoliberalismo en Michel Foucault. Buenos Aires: Biblioteca Universitaria, Ciencias Sociales y Humanidades. Filosofía Política.

Chávez Bidart, J. (2016). La psicología en la construcción de ciudadanía del Uruguay. Revista de Historia de la Psicología, (37), 23-31.

Córdoba, M. (2012). Políticas de la vida, retrato de una forma de vida emergente. Astrolabio, (8), 209-219.

Corea, C. y Lewkowicz, I. (1999) ¿Se acabó la infancia? Ensayo sobre la destitución de la niñez. Buenos Aires: Lumen.

Faraone, S., Barcala, A., Bianchi, E., \& Torriccelli, F. (2009). La industria farmacéutica en los procesos de medicalización/medicamentalización en la infancia. Revista Electrónica Margen,

Recuperado

de https://www.margen.org/suscri/margen54/faraone.pdf

Foucault, M. (2006). Seguridad, territorio y población. Buenos Aires: Fondo de Cultura Económica.

Foucault, M. (2008). La Vida de Los Hombres Infames. La Plata, Argentina: Altamira.

Germain, M. (2007). Elementos para una genealogía de la medicalización. Revista Cátedra Paralela, (4), 6-22.

Mussetta, P. (2009). Foucault y los anglofoucaultianos: una reseña del Estado y la gubernamentalidad. Revista Mexicana de Ciencias Políticas y Sociales, 51(205), 37-55. 
Pérez, R. (2010). La construcción subjetiva de realidad. Psicología, Neurociencias, Política e Imaginario Social. Conferencia inaugural. Actividades académicas 2009. Montevideo, Uruguay: Facultad de Psicología, Universidad de la República. Recuperado de http://www.redpsicogerontologia.net/images/Conferencia\%20Inaugural\%20200 9.pdf

Pinafi, T. (2013). Malestar y Psicofármacos: dependencia en la posmodernidad. Nómadas, $\quad$ (39), 79-89. $\quad$ Recuperado de http://www.redalyc.org/articulo.oa?id=105129195006

Rose, N. (1996). Una historia crítica de la psicología. En N. Rose. Inventing our Selves (Sandra De Luca y María del Carmen Marchesi, trad.). Cambridge: Cambridge University Press. Recuperado de http://www.elseminario.com.ar/biblioteca/Rose_Cap_2_Historia_critica_psicolog ia.htm

Rose, N. (2007). ¿La muerte de lo social?: Re-configuración del territorio de gobierno. Revista argentina de sociología, 5(8), 113-152.

Soneira, A. J. (2006). La teoría fundamentada en los datos (Grounded Theory) de Glaser y Strauss. En I. Vasilachis de Gialdino, Estrategias de investigación cualitativa (pp. 153-173). Barcelona: GEDISA.

Stolkiner, A. (2012). Infancia y medicalización en la era de "la salud perfecta". Buenos Aires, Argentina: Flacso.

Untoiglich, G. (2014). Medicalización y patologización de la vida: situación de las infancias en Latinoamérica. Nuances: estudios sobre Educación, 25(1). 20-38. Recuperado de: http://revista.fct.unesp.br/index.php/Nuances/article/viewFile/2743/2515

Valles, M. (1997). Técnicas cualitativas de investigación social. Reflexión metodológica y práctica profesional. Madrid, España: Síntesis. 
Vasilachis de Gialdino, I. (2006). Estrategias de investigación cualitativa. Barcelona: Gedisa.

Formato de citación

Chouhy, P. (2019). Las prácticas psicológicas en el ámbito escolar y su relación con el consumo de psicofármacos en la niñez. Psicología, Conocimiento y Sociedad, 9(1), 83-105. doi: http://dx.doi.org/10.26864/PCS.v9.n1.8 